\title{
The spacing effect in the learning of word pairs and the components of word pairs*
}

\author{
JOHN J. SHAUGHNESSY, JOEL ZIMMERMAN†, and BENTON J. UNDERWOOD \\ Northwestern University, Evanston, Illinois 60201
}

\begin{abstract}
Following the presentation of a single list of word pairs consisting of a three-letter word on the left and a five-letter word on the right, groups of $64 \mathrm{Ss}$ each were asked to recall the (a) three-letter words, (b) five-letter words, (c) intact pairs, or (d) five-letter words with the three-letter words provided. Two types of repeated pairs were presented, one in which the same three- and five-letter words were repeated together (same pairs) and one in which the same five-letter word was repeated with different three-letter words (different pairs). For half of the Ss in each recall group, the repetitions of a pair containing a given five-letter word were massed (MP); for the other half, the repetitions were distributed (DP). Recall of MP same pairs and the components of these pairs was consistently poorer than that of DP same pairs. Recall of the repeated component of the different pairs was also poorer under MP than under DP. The results were interpreted as supportive of an attenuation-of-attention explanation of the spacing effect.
\end{abstract}

One of the most consistent findings reported in the recent literature is that free recall of items occurring two or more times within a single trial is higher following a distributed practice schedule (DP) than following a massed practice schedule (MP). This difference has been referred to as the spacing effect. Hintzman's review (1974) of the evidence pertaining to the spacing effect indicates that no completely adequate theoretical account is available. Nevertheless, what has been referred to as the attenuation-of-attention hypothesis has been shown to have some explanatory value. Since certain conditions of the present experiment were viewed as representing tests of this hypothesis, the assumptions underlying the hypothesis will be stated explicitly.

The first assumption is that the total time law (i.e., that the amount learned is a direct function of effective study time) is correct. Since MP and DP items are exposed for the same nominal time but DP items are better recalled, it must follow that there is a greater discrepancy between nominal and effective study time for MP items than for DP items. Some support for this assumption can be found in studies which used self-paced study procedures in free recall (Zimmerman, 1973; Shaughnessy, Zimmerman, \& Underwood, 1972). These studies showed that Ss spent less time studying MP items than DP items. A substantial portion of the spacing effect in recall could be accounted for on the basis of the differences in exposure duration.

The second assumption is that attention reduction is not limited to the item being presented at the moment. There is assumed to be a commensurate reduction in the rehearsal of other items in the list during the interval in which the processing of the presented item is reduced.

\footnotetext{
*This research was supported by the Personnel and Training Research Programs, Psychological Sciences Division Office of Naval Research, under Contract N00014-67-A-0356-0010, Contract Authority Identification No. NR 154-321. Requests for reprints should be sent to Benton J. Underwood, Department of Psychology, Northwestern University, Evanston, Illinois 60201. †Presently at Social Research, Inc., Chicago, Hinois.
}

This assumption that attention reduction may reach a level where essentially all displaced rehearsal stops is suggested by the fact that the MP-DP differences in recall remain substantial even when unmixed lists are used (Underwood, 1969). If displaced rehearsal occurs effectively in a list consisting entirely of MP items, the overall recall should be as great as that for a list consisting entirely of DP items. In the study just cited, this was not the case.

The third assumption is that attenuation of attention is directly related to the degree of redundancy between the information being presented at the moment and the information available in memory. Information is more redundant for successive occurrences of MP items than for DP items, thus producing a larger discrepancy between nominal and effective study time for the former than for the latter. The present experiment can be viewed as a test of this third assumption.

The usual free-recall task, constituted of a list of single words, lacks flexibility when the desire is to vary redundancy. Therefore, we have turned to the paired-associate format. In particular, the interest was in studying the learning of MP pairs when the redundancy was varied. Consider three successively presented pairs in which, in one case, the pairs are identical $(A \cdot B, A-B$, $A-B)$ and, in the other, the left-hand terms differ (A-B, C-B, D-B). The two situations may be called same pairs and different pairs, respectively. The same pairs, when presented according to an MP schedule, represent the case of maximal redundancy, and it would be anticipated that the recall of the pairs or of the components of the pairs would be severely depressed as compared with the recall following a DP schedule. For the different pairs, the expectation changes. Redundancy is less for these pairs than for the same pairs, so learning should be better. The attenuation-of-attention hypothesis, however, as developed thus far, does not allow for a prediction as to whether the enhanced learning for the different pairs 
will generalize to both redundant and nonredundant components alike. For example, it is logically possible that the lack of redundancy among the left-hand terms in the different pairs could have absolutely no effect on the learning of the right-hand terms. It is even possible that the presence of the redundant right-hand terms may produce a deficit in the learning of the nonredundant left-hand terms. At this time, it is an empirical question as to what influence the presence of nonredundant components will have on the learning of redundant components, and vice versa.

The basic plan of the experiment, then, was to present same and different pairs under MP schedules to determine the influence of these manipulations on the learning of the components of the pairs as well as on the learning of the intact pairs. Corresponding conditions followed DP schedules because these schedules produce results which serve as reference values for the amount learned under conditions of minimal redundancy.

\section{METHOD}

\section{Lists}

Each $S$ was presented with a single list of word pairs which consisted of a total of 60 list positions. There were six pairs presented as a primacy buffer, six pairs as a recency buffer, eight pairs presented once each in the body of the list (singles), eight pairs presented twice each (Frequency 2), and eight pairs presented three times each (Frequency 3). All pairs consisted of a three-letter word on the left (stimulus) and a five-letter word on the right (response). The designation of the three-letter words as stimuli and the five-letter words as responses was done primarily for ease of reference. The Ss were not specifically instructed to treat the two classes differently; they were simply told to try to learn as many pairs as possible. For half of the pairs presented at Frequency 2 and Frequency 3, when a given response was repeated, it was paired with the same stimulus each time (same pairs). For the other half of the repeated pairs at both frequencies, when a given response was repeated, it was paired with a different stimulus each time (different pairs). Thus, a total of 48 stimuli and 36 responses was required to construct the list.

Forty-eight three-letter words were chosen to serve as stimuli and 36 five-letter words with Thorndike-Lorge frequencies of from 20 to 40 on the $\mathrm{G}$ count (Thorndike \& Lorge, 1944) were chosen to serve as responses. In both samples, words were chosen such that the first letters of the words were approximately equally distributed across the letters of the alphabet. In order to insure that a particular set of items was not confounded with a particular condition, four different random assignments of items to list functions were made. The assignments of items to these four forms were random, with the restrictions that no item could serve the same function in more than one form and that no two of the stimuli assigned to a given response in a given form could begin with the same first letter. This latter restriction applies, of course, only to the different pairs.

In order to avoid using prohibitively long lists, the MP-DP manipulation was carried out as a between-Ss manipulation. Each of the four forms just described was used once for the construction of an MP list and once for the construction of a DP list. After placing the primacy items in the first six positions and the recency items in the last six positions, the remaining 48 list positions were divided into four equal parts. Two singles were placed in each of the four list quarters, and these pairs occupied the same positions in both the MP and the DP lists. The repeated pairs were distributed approximately equally across the list quarters, although the exact list positions of given items obviously differed for the MP and DP lists. For both the MP and the DP lists, the positions occupied by same pairs in two of the forms were used for different pairs in the other two forms, and vice versa. In the DP lists, pairs were assigned to list positions such that both the position of last occurrence and the lag between repetitions were comparable for same pairs and different pairs.

\section{Procedure}

As the Ss arrived for the experimental session, they were assigned to one of five different recall conditions (by means of a seating assignment) according to a block randomized schedule. In the initial instructions read to the Ss, the exact nature of the memory test was not described, but all Ss were told that (a) they would be presented with a long list of pairs of words comprised of a three-letter word on the left and a five-letter word on the right; (b) they were to try to learn as many pairs as possible by associating the three-letter word to the five-letter word; (c) they would receive specific recall instructions immediately following the list presentation; (d) different Ss would be given different memory tests; (e) no Ss would be required to remember the order in which the pairs were presented; ( $f$ ) some of the pairs would be repeated; and (g) sometimes a given five-letter word would be repeated with different three-letter words. The Ss were asked subsequent to list presentation to recall stimulus terms (stimulus recall), response terms (response recall), intact pairs (pair recall), response terms with the stimulus terms provided (cued recall), or intact pairs with both the stimulus and response terms provided (matching).

The pairs were presented by means of a Kodak Carousel projector at a 5-sec rate. Immediately following the presentation of the last slide, Ss were given an instruction sheet appropriate to their recall condition. They were told to read the instructions, to ask any questions they might have individually, to begin to follow the instructions, and to continue trying to tecall until told to stop. All Ss were given $10 \mathrm{~min}$ for recall.

Ss in the stimulus-recall and response-recall groups were told that "thinking of" the other member of the pair might help them but that they were not restricted to this means of recalling the appropriate words. Ss in the pair-recall group were told that, if the same pair had occurred more than once, they needed to write it only once, but if the same five-letter word had been paired with different three-letter words, they were to write each of these pairings.

In the cued-recall group, Ss were given a list of all the stimulus words from the study list, randomly ordered, with those stimulus words that were presented more than once during study also appearing the same number of times on the cuing sheet. The repetitions of the stimulus words from the same pairs were included on the cuing sheet in an attempt to equate the number of opportunities for the recall of response terms included in same pairs and in different pairs. The repetitions were positioned randomly, with the restriction that the same stimulus word not occur in successive positions on the cuing sheet. In addition, six three-letter words were included on the sheet that had not been presented during study, with two of these words appearing once, two twice, and two three times. The Ss were told that the three-letter words were not listed in the same order as they had been presented during study and that some of the three-letter words on the sheet had not been presented. They were also told that, if a three-letter word appeared on the sheet more than once, they ware to write the appropriate five-letter word each time.

Ss in the matching group were given the same cuing sheet, as were Ss in the cued-recall group, and they were given an alphabetical listing of the response words. This list included some five-letter words (six) that were not presented during 
study, and the Ss were so instructed. It should be noted that the use of the alphabetical list of response words proved to be a cumbersome task for many Ss and that these Ss approached the matching task as if it were a cued-recall task. Because of this procedural difficulty, the data for the matching condition will not be presented.

\section{Design and Subjects}

There were 10 basic independent groups formed by the factorial combination of the MP-DP variable and the five types of recall tests. Thirty-two undergraduates were assigned to each of the 10 groups, for a total of $320 \mathrm{Ss}$. In addition, of the $32 \mathrm{Ss}$ in each group, groups of $8 \mathrm{Ss}$ were assigned to each of the four forms of the lists. The Ss participated in the experiment in groups of varying size, with all Ss within any experimental session receiving the same form of either the MP or the DP list but with the five types of recall being requested of an approximately equal number of Ss within each session.

\section{RESULTS}

The recall results will be presented in the following order. First, to determine the effect of the MP-DP variable on the same pairs and different pairs per se, the recall of the pair-recall group will be examined. Second, in order to assess the spacing effect on redundant pair members, the response-recall results will be presented. Third, an examination of the stimulus-recall results will provide an assessment of the spacing effect on the nonredundant components of the pairs. Finally, the results in the cued-recall group will allow for an assessment of the spacing effect in the recall of one component of a pair when the other component has been provided. For all analyses, values reported represent percentage values based upon a transformation of means obtained following an arc sine transformation of the raw score recall. Also, variation due to the four different forms of the MP and DP lists was accounted for in each analysis, but none of the effects of forms was evaluated.

\section{Pair Recall}

The scoring procedure used to evaluate the recall of pairs involved determining the percent recalled of a given type of pair, using as a base the total possible number of different pairs of that type. For the same pairs at Frequency 2 , each of four pairs was repeated intact twice and, as such, the total possible recall in this condition was four. For the same pairs at Frequency 3, the number possible was still four, with each pair having been presented three times. The situation changes for the different pairs. At Frequency 2, each of four responses was paired with a different stimulus on each repetition and, thus, the total number of possible pairs to be recalled was eight. Likewise, the total number of possible pairs at Frequency 3 was 12 . The mean percent recall, using this scoring procedure, for same and different pairs as a function of MP-DP and frequency is presented in the uppermost portion of Table 1. The mean percent recall of singles is also included in the table.

The recall of singles did not differ for the Ss assigned to MP lists (4.0\%) as compared to that for Ss assigned to DP lists $(2.8 \%), \mathrm{F}<1$. Analysis of the recall of repeated pairs indicated that the following effects were significant statistically $(\mathrm{p}<.01)$. It should be noted again that the percentage values reported for the various effects represent transformations of arc sine means. Therefore, it is not possible to obtain exactly the values reported by averaging those values presented in Table 1 . The resultant discrepancies, however, should be minor.

Table 1

Mean Percent Recall of Same and Different Pairs and Components of Pairs as a Function of Recall Instructions, MP-DP, and Frequency

\begin{tabular}{|c|c|c|c|c|c|c|c|}
\hline & & & \multirow[b]{2}{*}{ Singles } & \multicolumn{2}{|c|}{ Frequency 2} & \multicolumn{2}{|c|}{ Frequency 3} \\
\hline & & & & $\begin{array}{l}\text { Same } \\
\text { Pairs }\end{array}$ & $\begin{array}{c}\text { Different } \\
\text { Pairs }\end{array}$ & $\begin{array}{l}\text { Same } \\
\text { Pairs }\end{array}$ & $\begin{array}{c}\text { Different } \\
\text { Pairs }\end{array}$ \\
\hline \multirow[b]{2}{*}{ Pair Recall } & MP & $\begin{array}{l}\text { Mean } \\
\text { SD }\end{array}$ & $\begin{array}{c}4.0 \\
13.96\end{array}$ & $\begin{array}{c}6.3 \\
15.68\end{array}$ & $\begin{array}{c}4.4 \\
14.71\end{array}$ & $\begin{array}{l}15.2 \\
19.12\end{array}$ & $\begin{array}{c}8.6 \\
13.54\end{array}$ \\
\hline & DP & $\begin{array}{l}\text { Mean } \\
\text { SD }\end{array}$ & $\begin{array}{c}2.8 \\
11.89\end{array}$ & $\begin{array}{l}23.6 \\
17.56\end{array}$ & $\begin{array}{c}9.5 \\
15.82\end{array}$ & $\begin{array}{l}33.9 \\
22.73\end{array}$ & $\begin{array}{l}11.0 \\
13.38\end{array}$ \\
\hline \multirow{2}{*}{ Response Recall } & MP & $\begin{array}{l}\text { Mean } \\
\text { SD }\end{array}$ & $\begin{array}{c}6.8 \\
12.61\end{array}$ & $\begin{array}{l}13.5 \\
17.56\end{array}$ & $\begin{array}{l}10.3 \\
18.75\end{array}$ & $\begin{array}{c}8.4 \\
20.45\end{array}$ & $\begin{array}{l}16.4 \\
17.53\end{array}$ \\
\hline & DP & $\begin{array}{l}\text { Mean } \\
\text { SD }\end{array}$ & $\begin{array}{c}7.2 \\
14.28\end{array}$ & $\begin{array}{l}21.6 \\
19.88\end{array}$ & $\begin{array}{l}18.9 \\
21.62\end{array}$ & $\begin{array}{l}41.8 \\
20.35\end{array}$ & $\begin{array}{l}33.9 \\
19.40\end{array}$ \\
\hline \multirow{2}{*}{ Stimulus Recall } & MP & $\begin{array}{l}\text { Mean } \\
\text { SD }\end{array}$ & $\begin{array}{c}5.0 \\
14.78\end{array}$ & $\begin{array}{l}10.4 \\
18.75\end{array}$ & $\begin{array}{l}11.8 \\
12.72\end{array}$ & $\begin{array}{l}13.5 \\
17.96\end{array}$ & $\begin{array}{l}17.6 \\
11.37\end{array}$ \\
\hline & DP & $\begin{array}{l}\text { Mean } \\
\text { SD }\end{array}$ & $\begin{array}{l}11.4 \\
12.57\end{array}$ & $\begin{array}{l}35.5 \\
13.49\end{array}$ & $\begin{array}{l}17.6 \\
14.40\end{array}$ & $\begin{array}{l}46.7 \\
17.08\end{array}$ & $\begin{array}{l}21.6 \\
10.12\end{array}$ \\
\hline \multirow[b]{2}{*}{ Cued Recall } & MP & $\begin{array}{l}\text { Mean } \\
\text { SD }\end{array}$ & $\begin{array}{c}5.6 \\
16.28\end{array}$ & $\begin{array}{l}31.1 \\
24.77\end{array}$ & $\begin{array}{l}11.7 \\
17.89\end{array}$ & $\begin{array}{l}31.6 \\
24.94\end{array}$ & $\begin{array}{l}11.9 \\
16.37\end{array}$ \\
\hline & DP & $\begin{array}{l}\text { Mean } \\
\text { SD }\end{array}$ & $\begin{array}{l}11.0 \\
20.60\end{array}$ & $\begin{array}{l}45.9 \\
27.03\end{array}$ & $\begin{array}{l}22.5 \\
22.60\end{array}$ & $\begin{array}{l}70.6 \\
23.25\end{array}$ & $\begin{array}{l}22.1 \\
18.74\end{array}$ \\
\hline
\end{tabular}

Note-Percentage yalues are based upon a transformation of mean obtained following an arc sine transformation of the raw-score recall. Standard deviations are in arc sine units (degrees). 
(a) Overall, pairs repeated according to a DP schedule $(18.6 \%)$ were better recalled than those repeated according to an MP schedule $(8.2 \%), F(1,56)=8.61$. (b) Frequency-3 pairs $(16.2 \%)$ were better recalled than Frequency -2 pairs $(10.0 \%), F(1,56)=8.36$. (c) Same pairs $(18.6 \%)$ were better recalled than different pairs $(8.2 \%), \quad F(1,56)=27.37$. (d) Summing over the frequency variable, the MP-DP difference was greater for same pairs $(10.4 \%$ vs $28.6 \%)$ than for different pairs $(6.3 \%$ vs $10.2 \%), F(1,56)=7.64$. As can be seen in Table 1 , this interaction of the MP-DP and same-different variables did not differ for Frequency-2 as compared to Frequency-3 pairs. At both frequencies, the interaction of the MP-DP and same-different variables would seem to have been produced by the relatively high recall of DP same pairs.

In that the source of the interaction of the MP-DP and same-different variables is critical to the predictions derived from the attenuation-of-attention hypothesis, it was decided to perform Tukey's HSD test (cf. Keppel, 1973) on the pairwise comparisons of the four means comprising the interaction. The values are presented in Table 2. As indicated in the table, recall of DP same pairs was better than that of any of the other three types of pairs, which did not differ from each other. Support for the attenuation-of-attention hypothesis could be inferred from the fact that the recall of repeated intact pairs under MP (MP same pairs) was not better than that of the MP different pairs, which were presented only once each. Both of these types of pairs were more poorly recalled than intact pairs repeated under conditions assumed to minimize attenuation of attention (DP same pairs). The fact that DP different pairs were only slightly, and not significantly, better recalled than MP different pairs may be taken to indicate that, whatever attenuation of attention was produced by the presence of the single redundant component, the attenuation was not sufficient to influence the recall of the pairs.

\section{Response Recall}

The mean percent recall of response terms for singles and for same and different pairs as a function of MP-DP and frequency is presented in Table 1. The maximum possible recall for all repeated responses was four. As was the case in the recall of pairs, the mean percent of singles recalled by Ss assigned to the MP and DP groups did not differ, $\mathrm{F}<1$. For repeated responses, recall was better under DP $(28.6 \%)$ than under MP $(12.0 \%)$ $[F(1,56)=19.35, p<.01]$; recall was better for Frequency-3 responses (23.8\%) than for Frequency-2 responses $(15.8 \%)[\mathrm{F}(1,56)=5.35, \mathrm{p}<.05]$; and there was an interaction of the MP-DP and frequency variables such that the MP-DP difference was smaller for Frequency-2 responses $(11.8 \%$ vs $20.2 \%$ ) than for Frequency-3 responses $(12.1 \%$ vs $37.8 \%)$ $[F(1,56)=4.92, \mathrm{p}<.05]$.

The pairwise comparisons of the mean percent recall of responses from MP same, MP different, DP same, and
Table 2

Mean Percent Recall of MP and DP Same and Different Pairs and Components as a Function of Recall Instructions

\begin{tabular}{lcccc} 
& $\begin{array}{c}\text { MP } \\
\text { Same } \\
\text { Pairs }\end{array}$ & $\begin{array}{c}\text { MP } \\
\text { Different } \\
\text { Pairs }\end{array}$ & $\begin{array}{c}\text { DP } \\
\text { Different } \\
\text { Pairs }\end{array}$ & $\begin{array}{c}\text { DP } \\
\text { Same } \\
\text { Pairs }\end{array}$ \\
\hline Pair Recall & 10.4 & 6.3 & 10.2 & 28.6 \\
Response Recall & 10.8 & 13.2 & $\underline{26.2}$ & 31.2 \\
Stimulus Recall & 11.8 & 14.6 & 19.6 & 41.0 \\
\hline
\end{tabular}

Note-Underlined means are not significantly different from each other $(p>.05)$. For pair recall the critical range is $6.40 \mathrm{deg}$, for response recall $8.56 \mathrm{deg}$, and for stimulus recall $7.76 \mathrm{deg}$.

DP different pairs provide evidence concerning the presumed effect of attenuation of attention on the redundant component of these pairs. The relevant values are again presented in Table 2 . It is clear that the pattern of results for response recall is different from that present for pair recall. The responses repeated under DP were better recalled than those repeated under MP regardless of whether the response presented under MP was accompanied by a redundant (MP same) or nonredundant (MP different) component. In other words, the recall results lend support to the conclusion that the presence of a nonredundant component did not seem to reduce the attenuation of attention to the redundant component.

\section{Stimulus Recall}

The mean percent recall of stimulus terms for singles and for same and different pairs as a function of MP-DP and frequency is presented in Table 1 . The percentage values for stimuli from repeated pairs were determined the same way as was done for pair recall. Though the effect was small, the mean percent recall of singles for Ss assigned to DP lists (11.4\%) was better than that for Ss assigned to MP lists $(5.0 \%)[\mathrm{F}(1,56)=4.06, \mathrm{p}<.05]$. The pattern of results for the recall of stimulus terms from repeated pairs was almost identical to that for the recall of pairs per se. In general, the stimulus terms were better recalled than the pairs, but the effects of the MP-DP, frequency, and same-different variables were comparable to those found in pair recall. The recall of stimuli was better following DP $(29.8 \%)$ than MP $(13.2 \%)[\mathrm{F}(1,56)=29.11]$, better for Frequency 3 $(24.0 \%)$ than Frequency $2(18.0 \%) \quad[F(1,56)=8.28]$, and better for same pairs $(25.0 \%)$ than different pairs $(17.0 \%) \quad[F(1,56)=7.28]$. Also, summing over the frequency variable, the MP-DP difference was greater for same pairs (11.8\% vs $41.0 \%)$ than for different pairs $(14.6 \%$ vs $19.6 \%), F(1,56)=14.64$. All of these effects were significant at the .01 level.

As stated in the introduction, the effects of the presence of a redundant component on the recall of a nonredundant component could not be predicted from present statements of the attenuation-of-attention hypothesis. To assess these effects, pairwise comparisons 
comparable to those made for pair recall and response recall were again made for stimulus recall. The means for these comparisons involving the recall of stimuli are presented in Table 2. Again, the pattern of results is comparable to that found in pair recall. Stimuli from DP same pairs were better recalled than those from any of the other three types of pairs, which did not differ from each other. Evidence for the presumed effects of attenuation can be found in that repeated stimuli from MP same pairs were no better recalled than stimuli presented only once in MP different pairs. The fact that stimuli from MP different pairs (14.6\%) were slightly less well recalled than those from DP different pairs (19.6\%) might be taken as an indication that some of the attenuation to the redundant response terms under MP did affect the recall of the stimuli. The magnitude of the effect, however, is slight at best.

\section{Cued Recall}

The mean percent recall of response terms when stimulus terms were provided for singles and for same and different pairs as a function of MP.DP and frequency is presented in Table 1 . The percentage values for repeated pairs involve the same bases as were used for pair and stimulus recall. Recall of singles for those Ss assigned to DP lists $(11.0 \%)$ was not statistically significantly different from that for those assigned to MP lists $(5.6 \%) \quad[\mathrm{F}(1,56)=1.52, \mathrm{p}>.05]$. The only significant sources of variation in the analysis of the repeated pairs were the main effects of the MP-DP and same-different variables. Cued recall of DP pairs (39.6\%) was greater than that of MP pairs (20.8\%) $[\mathrm{F}(1,56)=9.38, \mathrm{p}<.01]$, and cued recall of same pairs $(44.8 \%)$ was greater than that of different pairs $(16.7 \%)$ $[\mathrm{F}(1,56)=82.86, \mathrm{p}<.01]$. As was the case in response recall, the MP-DP difference in cued recall was smaller for Frequency-2 pairs $(20.6 \%$ vs $33.7 \%)$ than for Frequency-3 pairs $(20.9 \%$ vs $45.8 \%)$, but this interaction was not significant statistically $[\mathrm{F}(1,56=2.40, \mathrm{p}>.05]$. Also, as was the case in pair recall and in stimulus recall, the MP-DP difference in cued recall was greater for same pairs $(31.4 \%$ vs $58.6 \%)$ than for different pairs $(11.8 \%$ vs $22.3 \%$ ), but, again, this interaction was not significant statistically $[F(1,56)=3.93, p>.05]$. Although the two interactions were not significant statistically, they might be taken as an indication that cued recall was influenced by factors affecting the recall of responses as well as by factors affecting the appropriate pairing of stimulus and response terms.

Comparing the pair-recall and cued-recall groups directly, it can be seen that recall for all types of pairs was better when one of the members of each pair was provided at the time of recall. For the MP same pairs, this increase was particularly striking, especially at Frequency 2. It would appear that, although attenuation of attention could be assumed to be present for the MP same pairs (as evidenced by the analysis of pair recall), Ss were able to recognize stimulus terms that they were not able to recall and to produce the appropriate response to these stimuli. A possible explanation for this finding, as well as for the effects found with the other types of recall, will be presented in the discussion.

\section{DISCUSSION}

Three issues will be considered in this section. First, the results will be summarized with an emphasis on how the findings are related to the third assumption of the attenuation-of-attention hypothesis that was presented in the introduction. Next, a more detailed explanation of these effects will be proposed, including a consideration of how it is that Ss may have gone about trying to learn the word pairs in the present experiment. Finally, the relationship of the findings of the present study to certain of the theories which have been proposed to account for the spacing effect in free recall will be discussed.

The present experiment was intended primarily as a test of the third assumption of the attenuation-of-attention hypothesis. This assumption, as stated in the introduction, is that attenuation of attention is directly related to the degree of redundancy between the information being presented at the moment and the information available in memory. The test of this assumption involved requiring Ss to learn pairs in which either both members of the pair provided redundant information (same pairs) or one member provided redundant information and one member was new (different pairs). It was anticipated that recall of same pairs repeated under MP would be poorer than that of same pairs repeated under DP. Also, it was expected that the recall of the components of same pairs would be poorer following MP than following DP. Both of these expectations were confirmed. An additional source of evidence for the assumption that attention deficits were present for the MP same pairs can be found by comparing the recall of these pairs with that of the MP different pairs. Recall of the repeated MP same pairs was not significantly better than the recall of the MP different pairs, each of which had been presented only once.

No predictions were made concerning the recall of the components of the different pairs or concerning the effects of providing access to one of the pair members at the time of recall (cued recall). It seemed equally plausible that the presence of a nonredundant component could enhance the recall of a redundant component or that the presence of a redundant component could inhibit the recall of a nonredundant component. In fact, neither of these effects was found. Recall of the redundant component of pairs including a nonredundant component (response recall of MP different pairs) was no better than that of the same component accompanied by a redundant component (response recall of MP same pairs). Likewise, the presence of the redundant component did not seem to be effective in lowering the recall of the nonredundant component (there was no difference in stimulus recall 
from MP different and DP different pairs). Finally, the cued-recall results indicated that providing access to one of the pair members at the time of recall enhanced recall for all pairs, but particularly for MP same pairs.

As stated in the introduction, the DP same and DP different pairs were assumed to provide conditions of repetition with minimal attenuation of attention. That this was the case is evidenced by the fact that recall increased for DP same pairs as compared to singles and for Frequency-3 DP same pairs as compared to Frequency-2 DP same pairs. Similar increases as a function of frequency were found for component and cued recall of DP same pairs. The repeated component of DP different pairs (the response) also showed an increase as a function of frequency. That the repetition of nominally identical events enhances the learning of these events is not surprising. What is surprising is that the beneficial effects of repetition are absent under certain conditions, namely, those assumed to involve attenuation of attention.

A possible explanation in terms of the attenuation-of-attention hypothesis will now be provided for the results summarized above. It should be emphasized, however, that this explanation has been suggested by the nature of the results rather than by any a priori assumptions of the attenuation-of-attention hypothesis. The present results seem to indicate that attenuation of attention can operate selectively. In order to allow for this selective attenuation, it would seem necessary to propose that Ss are faced with at least a twofold task when they are required to learn a list of word pairs. First, the Ss would need to establish a connection or association between the components of each of the pairs with the list context. This can be viewed as comparable to the response learning stage of the traditional two-stage theory of paired-associate learning (Underwood, Runquist, \& Schulz, 1959), but, of course, in the present situation both the stimuli and responses would need to be learned. The processes involved could be similar to those proposed by Bower (1972), whereby an item comes to be associated with an appropriate list marker, although the specifics of how the association to the list context is established are not of immediate concern. The second task facing Ss in the present situation would be finding some way to connect or associate the two components of a given pair to each other. This can be viewed as comparable to the associative stage of the traditional two-stage theory of paired-associate learning.

If it is plausible that these two tasks are involved in the learning of word pairs, then it would also seem reasonable to assume that, in a paced study procedure, Ss apportion the available time to these tasks in some systematic fashion. Recall of the components was better than the recall of the pairs following a single presentation. Any direct comparison of component and pair recall is difficult in that pair recall requires the joint recall of stimulus and response terms, while component recall requires the recall of only one of the components.
Nonetheless, the superiority of component recall might be taken as evidence that the initial presentation of a pair may have been devoted primarily to the learning of the components. Of course, the learning of the components and the learning of the association cannot be viewed as completely independent processes, and what is being considered here is the relative emphasis placed on these two processes during any given presentation. Associations between the components of a given pair were being established on the initial presentation, as evidenced by the fact that cued recall (based presumably on the association between pair members) was as good as the recall of either component following only a single presentation. The apportionment of study time in situations assumed to involve attenuation of attention (MP same and MP different) will now be considered.

On the initial presentation of an MP same pair, it is assumed that Ss spend more time learning the components of the pair and less time on the association between pair members. On the second and third presentations of an MP same pair, attention to the components is reduced, but time is spent on associating the pair members. It must be remembered that the attenuation of attention to the components is not assumed to be complete in that recall of MP same responses and stimuli was better than recall of singles. Also, the attention given to learning the association for MP same pairs is not assumed to be as great as that for DP same pairs in that recall of MP same pairs was consistently poorer than that of DP same pairs. The primary evidence for the differential attenuation of attention to the components and to the association between the pair members is that cued recall of MP same pairs showed such a marked increase over that of singles as compared to the increases over the singles found with response recall and stimulus recall.

The recall of the components of the MP different pairs provides another source of evidence for differential attenuation of attention. The second and third MP different pairs containing a given response term involved the introduction of one redundant component (response), one nonredundant component (stimulus), and a nonredundant association. The primary reduction in attention would be expected for the redundant response. The recall results are supportive of this position in that MP different responses were more poorly recalled than DP different responses.

Insofar as the procedures of the present experiment involved the paired-associate format, the relationship of the results to the situation involving the free recall of single items is not a direct one. Nonetheless, there is a relationship, especially when the results are viewed in terms of certain of the theories that have been proposed to account for the spacing effect in free recall. According to these theories (see Hintzman, 1974, for a review of specific theories), it is assumed that Ss encode the to-be-remembered word along several of a variety of dimensions. The resultant encodings are assumed to 
serve as recall cues for the to-be-remembered item. Thus, the storage and retrieval of to-be-remembered words implies the storage of compounds consisting of encodings (cues) and to-be-remembered items. If these compounds are at all analogous to the word pairs used in the present study, the results indicate that the mere presence of multiple encodings may not necessarily enhance the recall of the to-be-remembered item. The present procedures allow for an assessment of the effects of experimental manipulations on the components of compounds. In the typical situation involving the free recall of single items, the effects of these manipulations on the encodings of the to-be-remembered words must be inferred from the recall of the to-be-remembered items themselves.

\section{REFERENCES}

Bower, G. H. Stimulus-sampling theory of encoding variability, In A. W. Melton and E. Martin (Eds.), Coding processes in human memory. Washington, D.C: Winston, 1972. Pp. 85-123.

Hintzman, D. L. Theoretical implications of the spacing effect. Paper presented at the Loyola Symposium on Cognitive Psychology, Chicago, May 1973. To be published in R. L. Solso (Ed.), Theories of cognitive psychology: The Loyola symposium. Washing ton, D.C: Winston, 1974

Keppel, G. Design and analysis: A researcher's handbook. Englewood Cliffs, N.J: Prentice-Hall, 1973.

Shaughnessy, J. J., Zimmerman, J., \& Underwood, B. J. Further evidence on the MP-DP effect in free-recall learning. Journal of Verbal Learning \& Verbal Behavior, 1972, 11, 1-12.

Thorndike, E. L., \& Lorge, I. The teacher's word book of 30,000 words. New York: Columbia University Press, 1944.

Underwood, B. J. Some correlates of item repetition in free-recall learning. Journal of Verbal Learning \& Verbal Behavior, 1969, 8, 83-94.

Underwood, B. J., Runquist, W. N., \& Schulz, R. W. Response learning in paired-associate lists as a function of intralist similarity. Journal of Experimental Psychology, 1959, 58, 70-78.

Zimmerman, J. The effect of massed and distributed presentations in free-recall learning: $A$ test of the attenuation-of-attention hypothesis. Unpublished doctoral dissertation, Northwestern University, 1973.

(Received for publication March 13,1974; revision received April 24, 1974.) 